\title{
Frontières
}

\section{Alain Meunier et Gérard Tixier, Le grand blues. Faire face à la tentation du suicide chez un jeune, Paris, Payot, 2000, 246 pages}

\section{Éric Volant}

Volume 13, numéro 1, automne 2000

URI : https://id.erudit.org/iderudit/1074262ar

DOI : https://doi.org/10.7202/1074262ar

Aller au sommaire du numéro

Éditeur(s)

Université du Québec à Montréal

\section{ISSN}

1180-3479 (imprimé)

1916-0976 (numérique)

Découvrir la revue

Citer ce compte rendu

Volant, É. (2000). Compte rendu de [Alain Meunier et Gérard Tixier, Le grand blues. Faire face à la tentation du suicide chez un jeune, Paris, Payot, 2000,

246 pages]. Frontières, 13(1), 85-86. https://doi.org/10.7202/1074262ar d'utilisation que vous pouvez consulter en ligne.

https://apropos.erudit.org/fr/usagers/politique-dutilisation/ 
en deuil termine cette deuxième partie réservée à l'adulte ( $p$. 7277).

Le reste du livre est entièrement consacré au deuil des enfants. Les auteurs commencent par se demander ce que pense l'enfant de la mort, de celle des autres et de la sienne (p. 78-86). Bien sûr, les réponses divergent selon le développement intellectuel et la maturation pulsionnelle de chaque enfant. Toutefois, les enquêtes sont unanimes à conclure que les notions d'universalité et d'irréversibilité de la mort sont généralement bien acquises vers l'âge de 9 ans. En ce qui concerne le travail de deuil et son déroulement chez I'enfant (p. 87-101), il est le même que chez l'adulte. Les expressions de ces multiples étapes sont cependant assez différentes de celles des adultes. Évidemment, ces différences varient d'abord selon l'âge et le sexe de l'enfant au moment de la mort de son parent. Complexe d'Oedipe oblige... Parmi les différences signalées, les auteurs soulignent avec insistance celle qui a trait au comportement des enfants en deuil : ce comportement est à la fois calqué sur celui des proches et profondément influencé par ce qu'ils ressentent que ceux-ci attendent d'eux. Les auteurs proposent ensuite une brève réflexion sur l'avenir des enfants endeuillés (p. 102-111). Celui-ci dépend de plusieurs facteurs: I'état et l'âge de l'enfant au moment de la perte, la nature de la relation préexistante entre l'enfant et le parent qu'il a perdu, et la nature de l'accompagnement dont l'enfant a pu être entouré au cours de son deuil, aussi bien dans la famille qu'à l'école. Enfin, comme cette question de l'accompagnement est primordiale pour l'équilibre de l'enfant, l'ouvrage se termine par divers conseils qui sont adressés aussi bien aux membres de la famille qu'aux enseignants (p. 112-122). Parmi ceux-ci, mentionnons l'importance de l'écoute active (dire la vérité, aider à vivre ses émotions, à exprimer ses sentiments, etc.) et des cérémonies rituelles (autour des objets-souvenirs, par exemple, afin d'encourager les relations avec le souvenir de la personne qui est morte).

L'ouvrage se termine abruptement sans aucune conclusion. C'est dommage, car une synthèse finale aurait été bienvenue. Quant à la bibliographie (p. 123-125), elle n'inclut malheureusement pas plusieurs des noms cités dans le corps de l'ouvrage. En définitive, ce livre rendra d'excellents services à toute
Comprendre la mort et y réagir sont deux aspects très différents; ceci est particulièrement vrai chez le tout petit. Dans cet album on retrouve les deux aspects à la fois. Les auteures parlent de la réalité physique de la mort et des émotions que le décès suscite.

C'est là est un beau livre pour Dr Catherine Dolto-Tolitch et Colline Faure-Poirée

\section{Si on parlait de la mort}

Paris, Gallimard jeunesse/Giboulées, 1999, 12 pages.

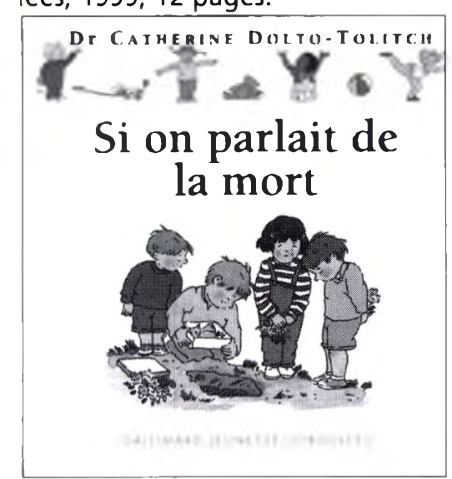

Cet album cartonné, illustré par Joëlle Boucher, s'adresse aux enfants d'âge préscolaire et aborde très succinctement divers sujets en lien avec la mort. La perte d'un être cher, les sentiments liés à ce départ et l'irréversibilité de la mort sont quelques-uns des thèmes prèsentés dans ce livre.

C. Dolto-Tolitch et C. FaurePoirée utilisent une approche directe. Par exemple, un dessin évoque le décès d'un enfant dans un accident de la route. Le discours qui l'accompagne invite l'enfant à parler des sentiments qui l'habitent.

Le jeune de cet àge qui est au coeur de la pensée magique, croit difficilement à la fin définitive de la vie. Ce n'est qu'à l'âge de six et même sept ans que l'enfant commence à comprendre la séparation qu'implique la mort. Tout petit, l'enfant ne saisit pas que tout le monde peut mourir et ce un peu n'importe quand. Pourtant, ce livre le confronte à cette vérité. On lui apprend que tous vont mourir parfois même ceux qu'il aime beaucoup.

Les auteures parlent à l'enfant de l'état du corps après la mort. Elles mentionnent au passage l'âme du défunt. Les dessins expriment la tristesse des parents et des adultes éprouvés. Les dernières images permettent d'insister sur l'importance du partage entre l'enfant et l'adulte pour parler de la peine que provoque la perte d'un être cher et pour échanger des souvenirs à son sujet. petit, sur un sujet qui peut grandement l'affecter tout en dépassant largement sa compréhension. Cependant, malgré son format et ses jolis dessins, je crois que cet album ne devrait pas se retrouver entre les mains d'un enfant sans la présence d'un adulte pour interpréter son contenu. Cette présence pourra lui permettre d'exprimer l'inquiétude et même l'angoisse que peuvent susciter certaines images. L'adulte pourra se servir de ce livre pour parler avec l'enfant d'un décès vécu. Il y retrouvera des idées pour mieux aider le jeune face à la mort. En définitive, cette lecture pourra alors devenir un prétexte pour permettre à l'enfant d'évoquer les questions, les peurs et tout ce qui le préoccupe.

\section{Sylvie Rhéaume}

Alain Meunier et Gérard Tixier

Le grand blues

Faire face à la tentation du suicide chez un jeune Paris, Payot, 2000, 246 pages.

ALAIN MEUNIER \& GERARD TIXIER

LEGRAND BLUES

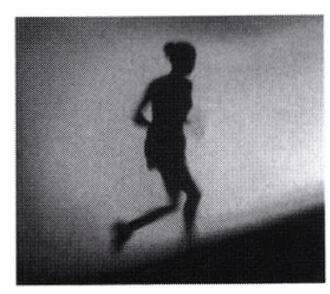

PAYOT

"Cent cinquante mille garçons et filles, âgés de quinze à vingtcinq ans, attentent à leurs jours en France chaque année. " (p. 11). Le rappel de ce fait brutal ouvre ce livre étonnant qui, soutenu par secondes avant leur fin, c'est ainsi que nous devons les voir : vivants, non pas comme des morts en puissance. [...] Ils se décrivent comme des êtres normaux, sains, animés d'une quête extrême, nous laissant à la rude tâche de les arrêter dans leur entreprise de destruction. " ( $p$. 13, " S'il est fou de mourir à vingt ans, le suicide n'est pas pour autant un signe de folie. " (p. 16). une écriture fort imagée, respire de vie et de santé. "Même trente

\section{Une longue expérience d'écoute}

Les deux auteurs se présentent comme " des psychiatres, psychothérapeutes et psychanalystes ", qui, durant quinze ans d'écoute téléphonique auprès des adolescents, ont dû composer constamment avec la mort. Celle-ci, chez les jeunes, " n'est plus un symptôme, mais un véritable vécu " (p. 13). Le but de ce livre est de révéler l'existence d'un "Mat Syndrome ", d'une sorte de trajectoire commune empruntée par les adolescents en marche vers la mort volontaire ; d'analyser les traits caractéristiques des cinq phases qui composent ce syndrome (chap. I) ; de décrypter les signaux de détresse que les adolescents lancent, tout au long de leur parcours, comme autant de bouteilles jetées à la mer (chap. II) ; de décrire la métamorphose que subit tout adolescent et le mal qui I'habite, de présenter les clés d'un dialogue qui empêche l'adulte et l'adolescent de sombrer dans leur solitude ; (chap. III) ; de proposer des façons d'intervenir auprès des jeunes adaptées à chacune des cinq phases de leur cheminement vers la mort (chap. IV)

\section{Les cinq phases}

du Mat Syndrome

"C'est pour échapper à une souffrance trop intense que les adolescents sont tentés par le suicide. La mort devient leur seul espoir, la seule issue possible. " ( $p$. 2 ). Afin de nommer cet itinéraire vers la mort, les auteurs inventent un nouveau terme " Mat Syndrome " qui vient de l'arabe mata qui signifie "être mort ". Ils ont pu détecter cinq phases dont chacune réfère à un moment critique du cheminement suicidaire et s'accompagne d'une nouvelle stratégie contre la souffrance. À l'origine, un accident initial se produit, une expérience intime et traumatique a lieu sous forme de vexation, rejet ou rupture. Afin de se protéger contre cette pénible réalité, l'adolescent se crée une bulle de rêve. C'est la phase de l'imaginaire-roi durant laquelle le jeune se réfugie dans un lieu ima- 
ginaire où il peut communiquer avec les autres et avec lui-même et où il peut penser librement à la mort sur un mode magique. Le recours à la mort, comme visa pour le pays de la fin heureuse, est d'abord une stratégie en faveur de la vie. Puis, vient le moment où le jeune s'aperçoit que la jolie mort se transforme en vraie mort. Perplexe et décontenancé, il entre dans la phase de lutte où, avec l'énergie du désespoir, il cherche à venir à bout de cette nécessité indésirable et à tuer la cause de ses tourments, à reconquérir la vie et à restaurer les moments heureux du passé. Cependant, sa mémoire est courte et le temps l'épuise. Alors, il se détourne de la réalité trop dure pour lui et il abandonne le combat. Il aborde la phase de renoncement où, avec tristesse et désarroi, il se soumet à son destin mortel. II n'est pas encore prêt à se tuer, mais il désire mourir ou être tué, tuer l'autre en soi, disparaître ou se désintégrer. Il aspire à la mort comme à " une sorte d'euthanasie » (p. 54), comme délivrance ou remède contre une insupportable souffrance. Puis approche l'heure où il décide de tuer le temps et de programmer sa propre fin. Il va jusqu'à préparer le scénario, repérer le lieu ou l'arme du crime. Le passage à l'acte devient une simple formalité. Cet état correspond à la phase du ressentiment où la toute-puissance est à son apogée et où, convaincu de l'inutilité de l'existence, il croit pouvoir maîtriser la mort pour en faire une œuvre d'art ou même pour entraîner autrui à le suivre. Enfin, dans la phase de l'œil du cyclone, l'adolescent vit dans un état de conscience altérée ou de transe. Il se sent comme déjà mort et il dispose d'un temps indéterminé pour se tuer. Cette indétermination peut être une porte de salut. La séquence des phases n'est donc pas irréversible. D'ailleurs, à chacune des phases, l'adolescent peut être aidé à trouver des repères extérieurs ou à puiser dans ses ressources afin de briser le linéaire de la mort.

\section{Le décryptage des signaux}

"En prêtant attention à ce que dit l'adolescent de la réalité, nous pouvons le situer sur sa trajectoire. " (p. 91). Le défi lancé à l'intervenant, c'est de saisir avec justesse le degré d'intensité des émotions du jeune qui se confie, la tonalité de son appel, les traits caractéristiques de son état de conscience, les particularités de sa perception d'autrui et de luimême, la nature de sa souffrance et la spécificité de son rapport à la mort (voir à cet effet les " tableaux récapitulatifs "à la fin du volume : p. 229-238). Si ce travail d'empathie est accompli avec le souci de la précision, l'intervenant peut savoir à quelle enseigne loge l'adolescent et, par conséquent, dire le mot juste et poser le geste adéquat. " Chaque phase constitue un moment particulier, un espace de vie, un univers en soi. [...] La prévention aura donc deux visages : limiter immédiatement la souffrance vécue et ménager dans la phase où l'adolescent se trouve un échange qui ouvre une dynamique de soins. " (p. 111).

\section{Le " psymal "}

\section{et la "pensée focale "}

Aux yeux des auteurs, Grand Bleu, film culte de Luc Besson est emblématique de la distance qui existe entre la culture des adolescents, qui y ont détecté un reflet de leur souffrance, et les adultes, qui ont éprouvé un malaise devant un film " sans scénario, sans intrigue, ni suspense ". On s'en doute, le choix du titre du présent volume est fortement inspiré par le titre du film. L'adolescence est marquée par une métamorphose physique et psychique. " L'adolescent est un être en mouvement et c'est ce mouvement qui le définit. [...] Ce n'est pas l'adolescent qu'il faut observer, mais sa trajectoire. "( $p$ 145). Cette métamorphose est faite d'émerveillement et de nouveauté, mais aussi de souffrance. Dans ce contexte, les auteurs reprennent la notion de "psymal ", développée par l'américain Edwin Schneidman dans Le tempérament suicidaire : "Le psymal se rapporte à la douleur, l'angoisse, le regret, la souffrance, la misère qui nous torturent l'esprit. C'est la souffrance intensément ressentie de la honte, de la culpabilité, de l'humiliation, de la solitude, de la perte, de la tristesse, de la terreur de vieillir ou de mal mourir, etc. Quand elle est ressentie, sa réalité introspective est indéniable. " (p. 160). Les auteurs élaborent aussi la notion de " pensée focale " qui fait qu'un individu concentre son intérêt sur un point unique de la réalité. Cette fixation change sa perception du reste du monde, comme elle le change aussi et lui fait oublier sa douleur. Chez l'adolescent suicidaire, l'objet de cette focalisation est la mort. " La trajectoire suicidaire est donc marquée par l'installation progressive d'une anesthésie psychique et physique facilitant le passage à l'acte. Les solutions préventives doivent passer par la mise en place d'un réveil, d'une sortie de la transe. " (p. 170).

\section{Que faire?}

Les auteurs proposent des stratégies sommaires d'intervention auprès des adolescents pour chacune des phases de l'itinéraire vers la mort. C'est sans doute la partie du présent volume qui risque de décevoir le plus les lecteurs. Une richesse inouie de connaissances sur le profil psychologique de l'adolescent hanté par l'idée de la mort aboutit à quelques conseils pratiques sans doute pertinents, mais si pauvres par rapport à la grande construction de l'esprit qui domine l'ensemble de ces pages. Ceci s'explique pourtant, car il y a une distance énorme, d'une part, entre l'idéal d'une écoute empathique et d'une action correspondante et, d'autre part, les incertitudes de nos observations et de nos interventions. En plus, cette construction logique, fondée sans doute dans la réalité observée et donc valable, fait penser aux stades du développement moral selon Lawrence Kohlberg ou aux étapes du deuil selon Élisabeth Kübler-Ross. Elle est révélatrice d'une évolution qui s'effectue par étapes et elle indique les balises d'une trajectoire. Elle suggère une voie sans autre prétention que celle d'une interprétation parmi d'autres de la mouvance de l'âme humaine à travers les aléas de l'existence, avec des avances et des reculs. Cette séquence, observée et présentée selon un ordre chronologique, ne doit donc pas être prise trop à la lettre, car elle risque d'enfermer l'adolescent en crise et l'intervention de l'adulte écoutant dans des cases trop étroites, plus ou moins validées et vérifiables. Cette réserve, exprimée avec la pudeur de celui qui ne sait pas trop, n'est qu'une interrogation critique que nous soumettons aux auteurs dont nous avons pu apprécier grandement l'approche empathique. Celle-ci naît d'une curiosité bienveillante ou d'un souci fondamental, celui de savoir ce qui se passe exactement dans l'esprit et le cœur de la jeune fille et du garçon aux prises avec l'idée ou le désir de la mort. Une toute dernière question sans doute déplacée : en fermant ce livre, on n'échappe pas à une certaine impression de dolorisme. Sans vouloir s'adonner à quelque minimalisme indécent, on peut se demander si la souffrance des adolescents est si généralisée. C'est à eux de nous le dire.

Éric Volant
André Compte-Sponville

Présentations de la philosophie

Paris, Albin Michel, 2000,

219 pages.

Andri Comte-Sponville Presentalions de la philosophice

albin Michel

Ce livre est la reprise de douze carnets indépendants et originellement publiés dans une collection d'initiation à la philosophie. Ces carnets, revus et sensiblement augmentès, constituent maintenant les douze chapitres du présent ouvrage. La modestie du propos reste toujours la même: il s'agit de douze portes d'entrée dans la philosophie. L'auteur a donc renoncé aux notes en bas de pages et a préféré commenter une brève bibliographie (p. 207-217). Ce choix est judicieux, car ces notes auraient alourdi considérablement l'ensemble de ses réflexions. En effet, plusieurs philosophes sont mentionnés à chaque page ou presque. Parmi les noms qui reviennent le plus souvent, on peut citer Kant (bien que Compte-Sponville affirme, à la page 139, ne pas être kantien), Spinoza, Montaigne et Alain, pour les Modernes, puis Platon et Aristote, pour les Anciens. En ce qui concerne les philosophes orientaux, seul Svâmi Prajnânpad, sage de I'Inde auquel Compte-Sponville a déjà consacré un livre, est cité à deux reprises (p. 183-184). Cette brève enquête sur les auteurs signalés par Compte-Sponville indique bien que la philosophie visée par le titre du livre est celle de l'Occident, ce qui ne présuppose aucunement, de l'aveu même de l'auteur, que la philosophie soit exclusivement grecque et occidentale (p. 15).

Les douze thèmes retenus sont les suivants: la morale, la politique, I'amour, la mort, la connaissance, la liberté, Dieu, l'athéisme, l'art, le 